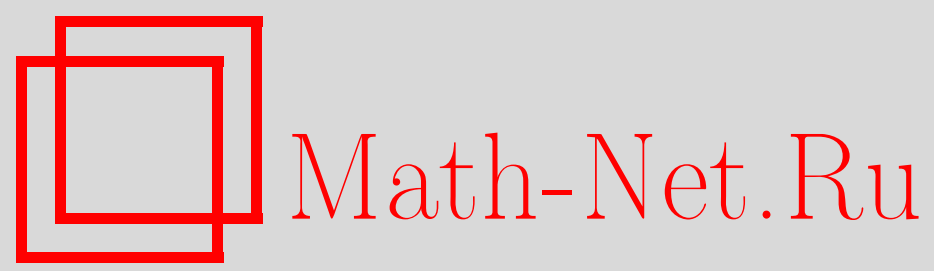

Д. А. Молодцов, Приближенные экстремумы ограниченного семейства интервалов, Нечеткие системы и мягкие вычисления, 2020, том 15, выпуск 2, 116-123

DOI: https://doi.org/10.26456/fssc73

Использование Общероссийского математического портала Math-Net.Ru подразумевает, что вы прочитали и согласны с пользовательским соглашением

http://www.mathnet.ru/rus/agreement

Параметры загрузки:

IP : 54.209 .52 .79

26 апреля 2023 г., 14:12:23 


\section{УДК 519.7 \\ ПРИБЛИЖЕННЫЕ ЭКСТРЕМУМЫ ОГРАНИЧЕННОГО СЕМЕЙСТВА ИНТЕРВАЛОВ}

Молодцов Д.А.

Вычислительный центр им. А.А. Дородницына Российской академии наук Федерального исследовательского центра «Информатика и управление» Российской академии наук, г. Москва

Поступила в редакчию 13.10.2020, после переработки 30.10.2020.

Устанавливаются связи между экстремальными множествами для "приближенньг” отношений доминирования для произвольного ограниченного семейства интервалов вещественных чисел.

Ключевые слова: «приближенное» доминирование интервалов, экстремальные множества интервалов.

Нечеткие системы и мягкие вычисления. 2020. Т. 15, № 2. С. 116-123. https://doi.org/10.26456/fssc73

\section{Введение}

Для ограниченного множества вещественных чисел верхняя грань этого множества может быть любым действительным числом, лишь бы это число было бы не менее нижней грани множества. Представляет интерес выяснить сохраняется ли аналогичное свойство для семейства интервалов вещественной оси. Прежде всего, на множестве интервалов возникает четыре естественных отношений порядка: больше, меньше, шире, уже. Для каждого из этих отношений доминирования можно ввести множество недоминируемых интервалов. Эти недоминируемые множества интервалов (которые будем называть экстремальными множествами) являются аналогами максимума и минимума для подмножества вещественных чисел. Для ограниченного и замкнутого семейства интервалов эти экстремальные множества уже связаны различными соотношениями [1]. В настоящей работе изучается та же проблематика, но свойство замкнутости семейства интервалов не предполагается, и рассматривается произвольное ограниченное семейство интервалов. Отсутствие замкнутости приводит к необходимости рассмотрения "приближенных" отношений доминирования, которые гарантируют непустоту экстремальных множеств для этих отношений. Для одного из возможных вариантов таких "приближенных" отношений устанавливаются связи между экстремальными множествами для произвольного ограниченного семейства интервалов.

\section{1. Основные обозначения}

Приведем список основных обозначений, операций и отношений, которые будут использованы далее. 
$R$ - множество действительных чисел, $R_{+}$- множество положительных действительных чисел. Под замкнутым интервалом $u$ множества действительных чисел понимается множество вида $u=\{x \in R \mid a \leq x \leq b\}$ для некоторых действительных чисел $a b \in R$ таких, что $a \leq b$. Обозначим $I R$ - множество замкнутых интервалов множества $R$.

Если $u \in I R$, то через $\underline{u}$ будем обозначать наименьшее число интервала $u$, а через $\bar{u}$ - наибольшее число интервала $u$. Таким образом, получаем $u=[\underline{u} \bar{u}]$.

Обозначения для основных отношений между двумя интервалами $u v \in I R$ ,$\varepsilon \in R_{+}$

$$
\begin{aligned}
& u>^{\varepsilon} v \Leftrightarrow \min \{\bar{u}-\bar{v}, \underline{u}-\underline{v}\} \geq 0 \wedge \max \{\bar{u}-\bar{v}, \underline{u}-\underline{v}\} \geq \varepsilon \\
& u<^{\varepsilon} v \Leftrightarrow \min \{\bar{v}-\bar{u}, \underline{v}-\underline{u}\} \geq 0 \wedge \max \{\bar{v}-\bar{u}, \underline{v}-\underline{u}\} \geq \varepsilon . \\
& u \supset^{\varepsilon} v \Leftrightarrow \min \{\bar{u}-\bar{v}, \underline{v}-\underline{u}\} \geq 0 \wedge \max \{\bar{u}-\bar{v}, \underline{v}-\underline{u}\} \geq \varepsilon \\
& u \subset^{\varepsilon} v \Leftrightarrow \min \{\bar{v}-\bar{u}, \underline{u}-\underline{v}\} \geq 0 \wedge \max \{\bar{v}-\bar{u}, \underline{u}-\underline{v}\} \geq \varepsilon
\end{aligned}
$$

Наряду с этими “строгими" отношениями будем использовать и "нестрогие” отношения:

$$
\begin{aligned}
& u \geq^{\varepsilon} v \Leftrightarrow\left\{u>^{\varepsilon} v \vee \max \{|\bar{u}-\bar{v}|,|\underline{u}-\underline{v}|\}<\varepsilon\right\} \\
& u \leq^{\varepsilon} v \Leftrightarrow\left\{u<^{\varepsilon} v \vee \max \{|\bar{u}-\bar{v}|,|\underline{u}-\underline{v}|\}<\varepsilon\right\} . \\
& u \supseteq^{\varepsilon} v \Leftrightarrow\left\{u \supset^{\varepsilon} v \vee \max \{|\bar{u}-\bar{v}|,|\underline{u}-\underline{v}|\}<\varepsilon\right\} \\
& u \subset^{\varepsilon} v \Leftrightarrow\left\{u \subset^{\varepsilon} v \vee \max \{|\bar{u}-\bar{v}|,|\underline{u}-\underline{v}|\}<\varepsilon\right\}
\end{aligned}
$$

\section{Утверждение 1.1.}

1. Отношения $>^{\varepsilon},<^{\varepsilon}, \supset^{\varepsilon}, \subset^{\varepsilon}$ транзитивны.

2. Пусть имеется цепочка одинаковых отношений $u_{1} \succ^{\varepsilon} u_{1} \succ^{\varepsilon} \ldots \succ^{\varepsilon} u_{n}$, где $\succ^{\varepsilon}$ везде обозначает одно из отношений $>^{\varepsilon},<^{\varepsilon}, \supset^{\varepsilon}, \subset^{\varepsilon}$. Тогда для $n=2 m$ выполнено $u_{1} \succ^{m \varepsilon} u_{n}$, а для $n=2 m+1$ выполнено $u_{1} \succ^{(m+1) \varepsilon} u_{n}$

3. Отношения $\geq^{\varepsilon}, \leq^{\varepsilon}, \supseteq^{\varepsilon} \subseteq^{\varepsilon}$ не являются транзитивными.

Утверждение 1.2. Пусть для двух интервалов иv $\in$ IR не справедливо ни одно из отношений

$$
u>^{\varepsilon} v, \quad u<^{\varepsilon} v \quad, \quad u \supset^{\varepsilon} v, \quad u \subset^{\varepsilon} v
$$

Тогда $\max \{|\bar{u}-\bar{v}|,|\underline{u}-\underline{v}|\}<\varepsilon$

Утверждение 1.3.

1. Пусть для двух интервалов $u v \in I R$ не справедливо ни одно из отношений

$$
u>^{\varepsilon} v, \quad u<^{\varepsilon} v
$$

Тогда справедливо одно из отношений $u \supseteq^{\varepsilon} v, u \subseteq^{\varepsilon} v$

2. Пусть для двух интервалов $u v \in I R$ не справедливо ни одно из отношений

$$
u \supset^{\varepsilon} v, \quad u \subset^{\varepsilon} v
$$

Тогда справедливо одно из отношений $u \geq^{\varepsilon} v, u \leq^{\varepsilon} v$

Подмножество (семейство) интервалов $\Xi \subseteq I R$ назовем ограниченным, если существует интервал $v \in I R$, что для любых интервалов $u \in \Xi$ выполнено $u \subseteq v$. Очевидно, подмножество ограниченного подмножества также ограничено. 
Пусть дана функция $f: X \rightarrow R$, где $X$ - некоторое множество и $\alpha \in R, \alpha \geq 0$. Будем использовать следующие обозначения для точек, где приближенно (или точно при $\alpha=0$ ) достигается верхняя и нижняя грани функции

$$
\begin{aligned}
& \operatorname{Argmax}(f, X, \alpha)=\{x \in X \mid f(x) \geq f(y)-\alpha\} \\
& \operatorname{Argmin}(f, X, \alpha)=\{x \in X \mid f(x) \leq f(y)+\alpha\}
\end{aligned}
$$

\section{2. Приближенно экстремальные множества семейства интервалов}

Для каждого из основных отношений введем множество недоминируемых элементов подмножества $\Xi$, которые будем называть приближенными экстремальными множествами семейства $\Xi$,

$$
\begin{aligned}
& \operatorname{Max}(\Xi, \varepsilon)=\left\{u \in \Xi \mid v \in \Xi: v>^{\varepsilon} u\right\} \\
& \operatorname{Min}(\Xi, \varepsilon)=\left\{u \in \Xi \mid v \in \Xi: v<^{\varepsilon} u\right\} \\
& \operatorname{Nar}(\Xi, \varepsilon)=\left\{u \in \Xi \mid v \in \Xi: v \subset^{\varepsilon} u\right\} \\
& \operatorname{Big}(\Xi, \varepsilon)=\left\{u \in \Xi \mid v \in \Xi: v \supset^{\varepsilon} u\right\}
\end{aligned}
$$

Утверждение 2.1. Пусть $\Xi \subseteq I R$ ограниченное подмножество интервалов и $\varepsilon>0$. Тогда

$$
\operatorname{Max}(\Xi, \varepsilon) \neq \emptyset, \quad \operatorname{Min}(\Xi, \varepsilon) \neq \emptyset, \quad \operatorname{Nar}(\Xi, \varepsilon) \neq \emptyset, \quad \operatorname{Big}(\Xi, \varepsilon) \neq \emptyset .
$$

Доказательство легко следует из утверждения 1.1.

Замечание. Для непустоты множества $\operatorname{Nar}(\Xi, \varepsilon)$ достаточно только условия $\varepsilon>0$.

Утверждение 2.2. Пусть $\Xi \subseteq I R$ ограниченное подмножество интервалов и $\varepsilon>0$.

1. Для любых интервалов $u v \in \operatorname{Max}(\Xi, \varepsilon)$ выполнено одно из отношений $u \supseteq^{\varepsilon} v, u \subseteq^{\varepsilon} v$

2. Для любых интервалов иv $\in$ Min $(\Xi, \varepsilon)$ выполнено одно из отношений $u \supseteq^{\varepsilon} v, u \subseteq^{\varepsilon} v$

3. Для любых интервалов иv $\in \operatorname{Nar}(\Xi, \varepsilon)$ выполнено одно из отношений $u \geq^{\varepsilon} v, u \leq^{\varepsilon} v$.

4.. Для любых интервалов uv $\in \operatorname{Big}(\Xi, \varepsilon)$ выполнено одно из отношений $u \geq^{\varepsilon} v, u \leq^{\varepsilon} v$.

Доказательство следует из утверждения 1.3.

Утверждение 2.3. Пусть $\Xi \subseteq I R$ ограниченное подмножество интервалов и $\alpha \varepsilon \in R, \varepsilon>\alpha$ Тогда

$$
\begin{gathered}
\operatorname{Max}(\Xi, \varepsilon) \supseteq \operatorname{Max}(\Xi, \alpha), \quad \operatorname{Min}(\Xi, \varepsilon) \supseteq \operatorname{Min}(\Xi, \alpha) \\
\operatorname{Nar}(\Xi, \varepsilon) \supseteq \operatorname{Nar}(\Xi, \alpha) \quad \operatorname{Big}(\Xi, \varepsilon) \supseteq \operatorname{Big}(\Xi, \alpha)
\end{gathered}
$$

Утверждение 2.4. Пусть $\Xi \subseteq I R$ ограниченное подмножество интервалов и $\varepsilon>0$.

1. Для любого $u \in \Xi$ Maх $(\Xi, \varepsilon)$ существует интервал $v \in \operatorname{Max}(\Xi, \varepsilon)$, такой что $v \geq^{\varepsilon} u$ 
2. Для любого $u \in \Xi$ Min $(\Xi, \varepsilon)$ существует интервал $v \in \operatorname{Min}(\Xi, \varepsilon)$, такой что $v \leq^{\varepsilon} u$

3. Для любого $u \in \Xi \operatorname{Nar}(\Xi, \varepsilon)$ существует интервал $v \in \operatorname{Nar}(\Xi, \varepsilon)$, такой чmo $v \subseteq^{\varepsilon} u$

4. Для любого $u \in \Xi$ Big $(\Xi, \varepsilon)$ существует интервал $v \in$ Big $(\Xi, \varepsilon)$, такой umo $v \supseteq^{\varepsilon} u$

Рассмотрим теперь экстремальные множества от экстремальных множеств.

Утверждение 2.5. Пусть $\Xi \subseteq I R$ ограниченное подмножеством интервалов и $\alpha \geq \varepsilon>0$. Тогда

$$
\begin{gathered}
\operatorname{Max}(\operatorname{Max}(\Xi, \varepsilon), \alpha)=\operatorname{Min}(\operatorname{Max}(\Xi, \varepsilon), \alpha)=\operatorname{Max}(\Xi, \varepsilon) \\
\operatorname{Min}(\operatorname{Min}(\Xi, \varepsilon), \alpha)=\operatorname{Max}(\operatorname{Min}(\Xi, \varepsilon), \alpha)=\operatorname{Min}(\Xi, \varepsilon) \\
\operatorname{Nar}(\operatorname{Nar}(\Xi, \varepsilon), \alpha)=\operatorname{Big}(\operatorname{Nar}(\Xi, \varepsilon), \alpha)=\operatorname{Nar}(\Xi, \varepsilon) \\
\operatorname{Big}(\operatorname{Big}(\Xi, \varepsilon), \alpha)=\operatorname{Nar}(\operatorname{Big}(\Xi, \varepsilon), \alpha)=\operatorname{Big}(\Xi, \varepsilon)
\end{gathered}
$$

Знаками $\bar{I}$ и $\underline{I}$ будем обозначать функции интервала, которые равны правому и левому концу интервала $\bar{I}(u)=\bar{u}, \underline{I}(u)=\underline{u}$.

Утверждение 2.6. Пусть $\Xi \subseteq I R$ ограниченное подмножество интервалов и $\delta>\alpha>\varepsilon>0$. Тогда
1. $\emptyset \neq \operatorname{Argmax}(\bar{I} \operatorname{Max}(\Xi, \varepsilon) \alpha) \cap \operatorname{Argmin}(\underline{\operatorname{I}}, \operatorname{Max}(\Xi, \varepsilon), \alpha) \subseteq \operatorname{Big}(\operatorname{Max}(\Xi, \varepsilon), \delta)$
2. $\emptyset \neq \operatorname{Argmin}(\bar{I} \operatorname{Max}(\Xi, \varepsilon) \alpha) \cap \operatorname{Argmax}(\underline{I}, \operatorname{Max}(\Xi, \varepsilon), \alpha) \subseteq \operatorname{Nar}(\operatorname{Max}(\Xi, \varepsilon), \delta)$
3. $\emptyset \neq \operatorname{Argmax}(\bar{I} \operatorname{Min}(\Xi, \varepsilon) \alpha) \cap \operatorname{Argmin}(\underline{I}, \operatorname{Min}(\Xi, \varepsilon), \alpha) \subseteq \operatorname{Big}(\operatorname{Min}(\Xi, \varepsilon), \delta)$
4. $\emptyset \neq \operatorname{Argmin}(\bar{I} \operatorname{Min}(\Xi, \varepsilon) \alpha) \cap \operatorname{Argmax}(\underline{I}, \operatorname{Min}(\Xi, \varepsilon), \alpha) \subseteq \operatorname{Nar}(\operatorname{Min}(\Xi, \varepsilon), \delta)$
$5 . \emptyset \neq \operatorname{Argmax}(\bar{I} \operatorname{Big}(\Xi, \varepsilon) \alpha) \cap \operatorname{Argmax}(\underline{I}, \operatorname{Big}(\Xi, \varepsilon), \alpha) \subseteq \operatorname{Max}(\operatorname{Big}(\Xi, \varepsilon), \delta)$
6. $\emptyset \neq \operatorname{Argmin}(\bar{I} \operatorname{Big}(\Xi, \varepsilon) \alpha) \cap \operatorname{Argmin}(\underline{I}, \operatorname{Big}(\Xi, \varepsilon), \alpha) \subseteq \operatorname{Min}(\operatorname{Big}(\Xi, \varepsilon), \delta)$
7. $\emptyset \neq \operatorname{Argmax}(\bar{I} \operatorname{Nar}(\Xi, \varepsilon) \alpha) \cap \operatorname{Argmax}(\underline{I}, \operatorname{Nar}(\Xi, \varepsilon), \alpha) \subseteq \operatorname{Max}(\operatorname{Nar}(\Xi, \varepsilon), \delta)$
$8 . \emptyset \neq \operatorname{Argmin}(\bar{I} \operatorname{Nar}(\Xi, \varepsilon) \alpha) \cap \operatorname{Argmin}(\underline{I}, N a r(\Xi, \varepsilon), \alpha) \subseteq \operatorname{Min}(\operatorname{Nar}(\Xi, \varepsilon), \delta)$
Доказательство. Докажем пункт 1. Остальные пункты доказываются аналогично.

Пусть $\beta \in R$ и $\beta=\alpha-\varepsilon>0$. Возьмем произвольный интервал $u \in \operatorname{Argmin}(\underline{\operatorname{I}}, \operatorname{Max}(\Xi, \varepsilon), \beta)$

Если $u \in \operatorname{Argmax}(\bar{I}, \operatorname{Max}(\Xi, \varepsilon), \alpha)$ то непустота пересечения

$$
\operatorname{Argmax}(\bar{I} \operatorname{Max}(\Xi, \varepsilon) \alpha) \cap \operatorname{Argmin}(\underline{\operatorname{I}}, \operatorname{Max}(\Xi, \varepsilon), \alpha)
$$

очевидна. Рассмотрим случай, когда $u \notin \operatorname{Argmax}(\bar{I}, \operatorname{Max}(\Xi, \varepsilon), \alpha)$, то есть

$$
u \in \operatorname{Max}(\Xi, \varepsilon) \quad \bar{u}<\bar{v}-\alpha
$$

Тогда возьмем произвольный интервал $w \in \operatorname{Argmax}(\bar{I}, \operatorname{Max}(\Xi, \varepsilon), \beta)$, то есть

$$
w \in \operatorname{Max}(\Xi, \varepsilon) \quad \bar{w} \geq \bar{v}-\beta
$$

Отсюда получаем справедливое неравенство

$$
\bar{u}<\bar{v}-\alpha \leq \bar{w}-\alpha+\beta=\bar{w}-\varepsilon
$$

то есть $\bar{w}-\bar{u}>\varepsilon$. 
Поскольку $u w \in \operatorname{Max}(\Xi, \varepsilon)$ то справедливы неравенства

$$
\begin{aligned}
& \min \{\bar{w}-\bar{u}, \underline{w}-\underline{u}\}<0 \vee \max \{\bar{w}-\bar{u}, \underline{w}-\underline{u}\}<\varepsilon \\
& \min \{\bar{u}-\bar{w}, \underline{u}-\underline{w}\}<0 \vee \max \{\bar{u}-\bar{w}, \underline{u}-\underline{w}\}<\varepsilon
\end{aligned}
$$

Отсюда следует $\underline{u}>\underline{w}$. Но из выбора интервала $u$ следует справедливость неравенства

$$
\underline{u} \leq \underline{v}+\beta
$$

Отсюда имеем

$$
\underline{w}<\underline{v}+\beta
$$

и, следовательно, $w \in \operatorname{Argmin}(\underline{\operatorname{I}}, \operatorname{Max}(\Xi, \varepsilon), \beta)$ Это означает, что

$$
\begin{gathered}
\operatorname{Argmax}(\bar{I}, \operatorname{Max}(\Xi, \varepsilon), \alpha) \cap \operatorname{Argmin}(\underline{I}, \operatorname{Max}(\Xi, \varepsilon), \alpha) \supseteq \\
\supseteq \operatorname{Argmax}(\bar{I}, \operatorname{Max}(\Xi, \varepsilon), \beta) \cap \operatorname{Argmin}(\underline{I}, \operatorname{Max}(\Xi, \varepsilon), \beta) \neq \emptyset .
\end{gathered}
$$

Предположим, что

$$
\operatorname{Argmax}(\bar{I} \operatorname{Max}(\Xi, \varepsilon) \alpha) \cap \operatorname{Argmin}(\underline{\operatorname{I}}, \operatorname{Max}(\Xi, \varepsilon), \alpha) \operatorname{Big}(\operatorname{Max}(\Xi, \varepsilon), \delta) \neq \emptyset .
$$

Возьмем теперь произвольный интервал

$$
p \in \operatorname{Argmax}(\bar{I}, \operatorname{Max}(\Xi, \varepsilon), \alpha) \cap \operatorname{Argmin}(\underline{\operatorname{I}}, \operatorname{Max}(\Xi, \varepsilon), \alpha) \operatorname{Big}(\operatorname{Max}(\Xi, \varepsilon), \delta) .
$$

Справедливы неравенства

$$
\underline{p} \leq \underline{v}+\alpha, \quad \bar{p} \geq \bar{v}-\alpha .
$$

Поскольку $p \notin \operatorname{Big}(\operatorname{Max}(\Xi, \varepsilon), \delta)$, то существует интервал $q \in \operatorname{Max}(\Xi, \varepsilon)$ такой, что

$$
\min \{\bar{q}-\bar{p}, \underline{p}-\underline{q}\} \geq 0 \wedge \max \{\bar{q}-\bar{p}, \underline{p}-\underline{q}\} \geq \delta
$$

Отсюда получаем либо $\bar{q} \geq \bar{p}+\delta$ и тогда

$$
\bar{q} \geq \bar{p}+\delta \geq \bar{v}+\delta-\alpha
$$

противоречие, либо $\underline{q} \leq \underline{p}-\delta$ и тогда

$$
\underline{q} \leq \underline{p}-\delta \leq \underline{v}-\delta+\alpha
$$

опять противоречие. ?

Утверждение 2.7. Пусть $\Xi \subseteq I R$ ограниченное подмножество интервалов $u \varepsilon>\alpha>0$. Тогда

1. $\operatorname{Argmax}(\bar{I}+\underline{I}, \Xi, \alpha) \subseteq \operatorname{Max}(\Xi, \varepsilon)$.

2. $\operatorname{Argmax}(\bar{I}-\underline{I}, \Xi, \alpha) \subseteq \operatorname{Big}(\Xi, \varepsilon)$.

3. $\operatorname{Argmin}(\bar{I}+\underline{I}, \Xi, \alpha) \subseteq \operatorname{Min}(\Xi, \varepsilon)$.

4. $\operatorname{Argmin}(\bar{I}-\underline{I}, \Xi, \alpha) \subseteq \operatorname{Nar}(\Xi, \varepsilon)$.

Доказательство. Докажем пункт 1. Возьмем произвольный интервал $v \in \operatorname{Argmax}(\bar{I}+\underline{I}, \Xi, \alpha)$ Предположим, что $v \notin \operatorname{Max}(\Xi, \varepsilon)$ Тогда существует интервал $u \in \Xi$ такой, что

$$
\min \{\bar{u}-\bar{v}, \underline{u}-\underline{v}\} \geq 0 \wedge \max \{\bar{u}-\bar{v}, \underline{u}-\underline{v}\} \geq \varepsilon
$$


Поскольку справедливо неравенство $\bar{v}+\underline{v} \geq(\bar{w}+\underline{w})-\alpha \geq \bar{u}+\underline{u}-\alpha$ то отсюда следуют неравенства $\alpha \geq \bar{u}-\bar{v}+\underline{u}-\bar{v} \geq \max \{\bar{u}-\bar{v}, \underline{u}-\underline{v}\} \geq \varepsilon$. Противоречие.

Пункт 2. Возьмем $v \in \bar{\epsilon} \operatorname{Argmax}(\bar{I}-\underline{I}, \Xi, \alpha)$ Предположим, что $v \notin \operatorname{Big}(\Xi, \varepsilon)$ Тогда существует интервал $u \in \Xi$ такой, что выполнены неравенства

$$
\min \{\bar{u}-\bar{v}, \underline{v}-\underline{u}\} \geq 0 \wedge \max \{\bar{u}-\bar{v}, \underline{v}-\underline{u}\} \geq \varepsilon
$$

Поскольку справедливо неравенство $\bar{v}-\underline{v} \geq(\bar{w}-\underline{w})-\alpha \geq \bar{u}-\underline{u}-\alpha$ то отсюда следуют неравенства $\alpha \geq \bar{u}-\bar{v}+\underline{v}-\underline{u} \geq \max \{\bar{u}-\bar{v}, \underline{v}-\underline{u}\} \geq \varepsilon$. Противоречие.

Остальные пункты доказываются аналогично. ?

Утверждение 2.8. Пусть $\Xi \subseteq I R$ ограниченное подмножество интервалов $u \varepsilon>$. Тогда
1. $\bar{u}=\bar{u}=\bar{u}$.
2. $\underline{u}=\underline{u}=\underline{u}$.
3. $\underline{u}=\underline{u}=\underline{u}$.
4. $\bar{u}=\bar{u}=\bar{u}$.

Доказательство. Докажем пункт 1. Доказательство остальных пунктов аналогично. Неравенство

$$
\bar{u} \geq \bar{u}
$$

--очевидно. Предположим, что

$$
\bar{u}>\bar{u}
$$

Тогда существует интервал $v \in \Xi$ такой, что

$$
\bar{u} \geq \bar{v}>\bar{u}
$$

Поскольку $v \notin \operatorname{Max}(\Xi, \varepsilon)$, то существует интервал $w \in \operatorname{Max}(\Xi, \varepsilon)$ такой, что

$$
\min \{\bar{w}-\bar{v}, \underline{w}-\underline{v}\} \geq 0 \wedge \max \{\bar{w}-\bar{v}, \underline{w}-\underline{v}\} \geq \varepsilon
$$

Отсюда получаем $\bar{w} \geq \bar{v}>\bar{u}$ - противоречие, следовательно

$$
\bar{u}=\bar{u}
$$

Равенство

$$
\bar{u}=\bar{u}
$$

доказывается аналогично.?

Утверждение 2.9. Пусть $\Xi \subseteq I R$ ограниченное подмножество интервалов $u \varepsilon>$.

1. Для любых $u \in \operatorname{Max}(\Xi, \varepsilon)$ и любых $v \in \operatorname{Big}(\Xi, \varepsilon)$ выполнено неравенство $\underline{u}+\varepsilon>\underline{v}$

2. Для любых $u \in \operatorname{Min}(\Xi, \varepsilon)$ и любых $v \in \operatorname{Big}(\Xi, \varepsilon)$ выполнено неравенство $\bar{v}+\varepsilon>\bar{u}$

3. Для любых $u \in \operatorname{Max}(\Xi, \varepsilon)$ и любых $v \in \operatorname{Nar}(\Xi, \varepsilon)$ выполнено неравенство $\bar{u}+\varepsilon>\bar{v}$

4. Для любых $u \in \operatorname{Min}(\Xi, \varepsilon)$ и любых $v \in \operatorname{Nar}(\Xi, \varepsilon)$ выполнено неравенcmвo $\underline{u}+\varepsilon>\underline{v}$

Доказательство. Докажем пункт 3. Остальные пункты доказываются аналогично. Предположим, что выполнено неравенство $\bar{u}+\varepsilon \leq \bar{v}$. Если $\underline{v} \geq \underline{u}$, то $v>^{\varepsilon} u$ и это означает, что $u \notin \operatorname{Max}(\Xi, \varepsilon)$. Если $\underline{v}<\underline{u}$, то $u \subset^{\varepsilon} v$ и это означает, что $v \notin \operatorname{Nar}(\Xi, \varepsilon)$,противоречие, следовательно, справедливо неравенство $\bar{u}+\varepsilon>\bar{v} . ?$ 


\section{Заключение}

Для произвольного ограниченного семейства интервалов были введены экстремальные множества для "приближенных" отношений доминирования, построенных на базе естественных отношений для интервалов: больше, меньше, шире, уже. Установлены связи между этими экстремальными множествами, а также между экстремальными множествами от экстремальных множеств. Особо отметим утверждение 2.6., где установлена возможность одновременной максимизации (минимизации) правого и левого конца интервала по одному из экстремальных множеств, причем результат такой одновременной оптимизации дает подмножество соответствующего экстремального множества от экстремального множества, по которому проводилась оптимизация. Полученные результаты могут быть полезны для оптимизации функций с интервальными значениями.

\section{Список литературы}

[1] Молодцов Д.А. Экстремальные множества семейства интервалов // Нечеткие системы и мягкие вычисления. 2018. Т. 13, № 1. С. 5-15.

\section{Образец цитирования}

Молодцов Д.А. Приближенные экстремумы ограниченного семейства интервалов // Нечеткие системы и мягкие вычисления. 2020. Т. 15, № 2. С. 116-123. https://doi.org/10.26456/fssc73

\section{Сведения об авторах}

\section{1. Молодцов Дмитрий Анатольевич}

ведущий научный сотрудник Вычислительного центра им. А.А. Дородницына Российской академии наук Федерального исследовательского центра «Информатика и управление» Российской академии наук.

Россия, 119311, г. Москва, ул. Вавилова, д. 40, ВЦ РАН. 


\title{
APPROXIMATE EXTREMA OF A BOUNDED FAMILY OF INTERVALS
}

\author{
Molodtsov Dmitriy Anatolievich
}

Leading Researcher at Institution of Russian Academy of Sciences, Dorodnicyn Computing Centre of RAS

Russia, 119333, Moscow, 40 Vavilova str., CC RAS.

Received 13.10.2020, revised 30.10.2020

Connections are established between extremal sets for "approximate" dominance relations for an arbitrary bounded family of intervals of real numbers.

Keywords: "approximate" domination of intervals, extreme sets of intervals.

\section{Citation}

Molodtsov D.A., "Approximate extrema of a bounded family of intervals", Nechetkie Sistemy i Myagkie Vychisleniya [Fuzzy Systems and Soft Computing], 15:2 (2020), 116-123(in Russian). https://doi.org/10.26456/fssc73

\section{References}

[1] Molodtsov D.A., "Extremal sets of the family of intervals", Nechetkie Sistemy i Myagkie Vychisleniya [Fuzzy Systems and Soft Computing], 13:1 (2018), 5-15 (in Russian). 